\title{
Arm cavity resonant sideband control for laser interferometric gravitational wave detectors
}

\author{
D. E. McClelland \\ Department of Physics, The Faculties, The Australian National University, Science Road, Canberra 0200, Australia \\ J. B. Camp, J. Mason, W. Kells, and S. E. Whitcomb \\ LIGO Project, California Institute of Technology, MS 51-33, 1200 East California Boulevard, Pasadena, California 91125
}

Received May 3, 1999

\begin{abstract}
We present a new optical control scheme for a laser interferometric gravitational wave detector that has a high degree of tolerance to interferometer spatial distortions and noise on the input light. The scheme involves resonating the $\mathrm{rf}$ sidebands in an interferometer arm cavity. (c) 1999 Optical Society of America

OCIS codes: $120.3180,120.2230$.
\end{abstract}

The scientific goal of direct measurement of gravitational radiation will be pursued in the next decade with the use of laser interferometry. Long-baseline interferometers are under construction at a number of locations $\mathrm{s}^{1,2}$ and are expected to become operational in the next few years. In such first-generation instruments, light from a frequency and amplitude stabilized laser is injected into an interferometer configured as a recycled Michelson interferometer with Fabry-Perot arm cavities; $r f$ sideband phase modulation techniques are used to provide a phase reference for measuring small differential length changes and to extract length control signals. ${ }^{3}$ The sidebands and the carrier undergo different filterings in the interferometer as they propagate to the gravitational wave (gw) signal port, where length changes of the arm cavities that are due to a gravitational wave may be observed.

The interferometer sensitivity is directly related to the spatial and temporal stability of the carrier and sideband frequencies. In the control scheme employed by the Laser Interferometer Gravitational Wave Observatory $^{1}$ (LIGO) and VIRGO, ${ }^{2}$ which we will call the singly resonant sideband scheme (SRS), the rf sidebands propagate only in the nearly degenerate power recycling cavity (PRC), whereas the carrier is resonant in both the PRC and the arm cavities. Therefore, as explained below, the carrier receives spatial and temporal filtering that the sidebands do not receive. As a result, most gw signal noise sources related to the laser light are dominated by sideband noise, instability, or both. These sources include thermal lensing from absorption in the recycling cavity, ${ }^{4} \mathrm{rf}$ intensity noise,${ }^{5}$ and in-band frequency and intensity noise. ${ }^{6}$

We consider here an alternative optical control scheme that would reduce the coupling of all the above noise terms to the gw signal output. The new approach involves resonating the $\mathrm{rf}$ sidebands in an arm cavity. This achieves spatial and temporal filtering of the sidebands, thus providing a more robust, stable configuration.

For the analysis presented here we use the nominal LIGO parameters: recycling mirror transmission and radius of curvature, $3 \%$ and $10 \mathrm{~km}$, respectively; in- put test mass transmission, 3\%; optic loss, 50 parts in $10^{6}$. For the SRS system a Michelson asymmetry $(\delta)$ of $0.4 \mathrm{~m}$ is chosen to permit maximum transmission of the $25-\mathrm{MHz}$ sideband frequency to the gw signal port. As shown in Fig. 1, four length degrees of freedom (dof's) need to be controlled about their nominal values: the arm cavity and recycling cavity common mode lengths, $L_{+}=L_{x}+L_{y}$ and $l_{+}=l_{x}+l_{y}$, and the arm cavity and recycling cavity differential mode lengths, $L_{-}=L_{x}-L_{y}$ and $l_{-}=l_{x}-l_{y}=\delta$, respectively. Setting $l_{+}=(m+1 / 2) c / f_{\mathrm{sb}}$, where $m$ is an integer and $f_{\mathrm{sb}}$ is the sideband frequency, ensures that when the carrier resonates in the arms and in the recycling cavity the sidebands will resonate only in the recycling cavity. Control signals are obtained when cavity length deviations from resonance cause differential phase shifts of the carrier relative to the $\mathrm{rf}$ sidebands that interfere at the photodetectors as a beating at the rf. Demodulation of the detector output into in-phase and quadrature-phase components relative to the modulation phase then gives cavity length control signals.

Extensive analysis has been carried out on the standard SRS scheme. Apparent gw signal noise caused

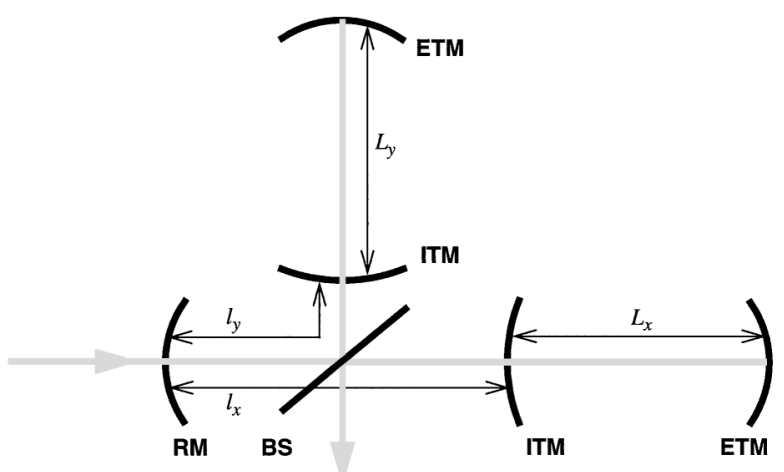

Fig. 1. Schematic layout of the LIGO interferometer: ITM's, input test masses; ETM's, end test masses; $\mathrm{BS}$, beam splitter; RM, recycling mirror. The lengths $l_{x}$, $l_{y}, L_{x}$, and $L_{y}$ are controlled. Light is shown exiting the interferometer at the gw signal port. 
by coupling of laser and oscillator noise to arm cavity rms length fluctuations $\left(\sim 10^{-13} \mathrm{~m}\right)$ and imperfect matching of the interferometer arm reflectivities $(\sim 0.5 \%)$ set requirements on the laser and oscillator stability. Column 2 of Table 1 summarizes the requirements for LIGO to achieve a sensitivity of $10^{-20} \mathrm{~m} / \mathrm{Hz}^{1 / 2}$ at $100 \mathrm{~Hz}^{6}{ }^{6}$ Fluctuations at the gw signal port arise because of beating of the rf sidebands with audio band noise of the carrier and beating of carrier light with audio band noise on the rf components. ${ }^{6}$ As the carrier light is resonant in both the recycling cavity and the arm cavities, it propagates in a coupled cavity with a linewidth given by $f_{\text {cc }}=$ $f_{\mathrm{c}}\left(1+r_{r} * r_{0}\right) / 2$, where $f_{\mathrm{c}}$ is the arm cavity linewidth, $r_{r}$ and $r_{0}$ are the recycling mirror and arm cavity amplitude reflectivities for the carrier, respectively, and $f_{\mathrm{cc}}$ is the coupled-cavity linewidth. This formula gives a half-linewidth of $\sim 1 \mathrm{~Hz}$. As a result, audio band noise on the carrier light above $1 \mathrm{~Hz}$ is attenuated. The $\mathrm{rf}$ sidebands, however, are not resonant in the arm cavities; they propagate only in the recycling cavity, which has a linewidth of $75 \mathrm{kHz}$. Hence in the SRS scheme it is the audio band noise on the rf sidebands beating with the carrier light that dominates the noise budget.

In the initial phase of the LIGO, $6 \mathrm{~W}$ of laser power at $1.06-\mu \mathrm{m}$ wavelength will be incident upon the powerrecycling mirror. Measurements of the optics put the absorption in the input test mass and beam splitter substrates at the order of 5 parts in $10^{6}$ per centimeter and coating absorption at 0.5 part in $10^{6}$. Absorption in the input test mass induces a transverse and longitudinal spatial variation in the refractive index of the fused silica, which then acts as a lens on the propagating laser beam. This phenomenon, referred to as thermal lensing, ${ }^{4}$ alters the wave-front curvature of the beam so that it no longer matches the curvature of the recycling cavity. As the recycling cavity is almost degenerate $\left(g_{1} g_{2}=0.997\right)$ and highly susceptible to wave-front errors, the result is degraded power buildup and transmission of sidebands. This effect has been simulated with a computer model of the interferometer that uses a fast Fourier transform- (FFT-) based optical propagation code, ${ }^{7}$ including the surface figure of all optical components and optical path-length difference maps of substrates with finite absorption. As a measure of the effect of thermal lensing, we consider the sideband power buildup in the recycling cavity, given by the recycling gain $\left(G_{\mathrm{sb}}\right)$, which we optimize by varying the Michelson asymmetry. For the nominal parameters of the LIGO, $G_{\mathrm{sb}}$ is $\sim 30$ in the absence of thermal lensing. As shown in Fig. 2, the inclusion of thermal lensing in the input test mass yields a sideband gain of less than 2 for the nominal recycling mirror radius of curvature $(10 \mathrm{~km})$. This result presents severe difficulties for both interferometer control and signal extraction and cannot be compensated for by a simple increase in the modulation depth. Figure 2 also shows that it is possible to recover most of the circulating power if the curvature of the recycling mirror can be altered to compensate for the distorted field. This approach, however, is optimal for a restricted range of laser power and recycling mirror curvature. We note that a curvature variation of 5\% from this value causes a further drop in $G_{\mathrm{sb}}$ of $20 \%$. We also find that a change of input power of just $\pm 1 \mathrm{~W}$ results in a $40 \%$ change in $G_{\mathrm{sb}}$, accompanied by a $10 \%$ decrease in the interferometer strain sensitivity, after the modulation depth is optimized.

It is important to appreciate that the carrier field resonant buildup is not subject to these effects. ${ }^{7}$ The carrier resonance in the nondegenerate arm cavities $\left(g_{1} g_{2} \sim 0.3\right)$ effectively stabilizes its $\mathrm{TEM}_{00}$ mode such that the net double resonance is highly stable. Extensive FFT modeling shows this clearly: For the parameters of Fig. 2, the carrier recycling gain varies by less than $2 \%$ in the presence of input test mass thermal lensing. A modal picture of the mechanism for this is evident: The overcoupled arm cavities select the desired resonant mode $\left(\mathrm{TEM}_{00}\right)$ by introducing a $\pi$ phase shift on the reflected wave. All other non-armresonant modes are then antiresonant in the doublecavity system. In contrast, at the sideband frequency no special mode is selected by resonance in the arms, so all modes resonate in the recycling cavity, leading to a corresponding loss of power from the $\mathrm{TEM}_{00}$ mode.

Extending the arm cavity mode selectivity to the sideband light renders the rf sidebands similarly immune to spatial distortions. To do this we allow the rf sideband pair to resonate in one of the arm cavities (allowing the rf sidebands to resonate in both arm cavities $^{8}$ will not provide enough signals for length control) and set $l_{+}=(m+1 / 2) c / 2 f_{\mathrm{sb}}$. We refer to this

Table 1. Noise Requirements at $100 \mathrm{~Hz}$ for $10^{-20} \mathrm{~m} / \mathrm{Hz}^{1 / 2} \mathrm{gw}$ Sensitivity

\begin{tabular}{lcc}
\hline Noise & SRS Value & SOAS Value \\
\hline $\begin{array}{l}\text { Laser } \\
\text { frequency }\end{array}$ & $2 \times 10^{-7} \mathrm{~Hz} / \mathrm{Hz}^{1 / 2}$ & $6 \times 10^{-6} \mathrm{~Hz} / \mathrm{Hz}^{1 / 2}$ \\
$\begin{array}{c}\text { Laser } \\
\text { amplitude }\end{array}$ & $10^{-7} / \mathrm{Hz}^{1 / 2}$ & $3 \times 10^{-6} / \mathrm{Hz}^{1 / 2}$ \\
$\begin{array}{c}\text { Laser rf } \\
\text { amplitude }\end{array}$ & $8 \times 10^{-10} / \mathrm{Hz}^{1 / 2}$ & $2 \times 10^{-8} / \mathrm{Hz}^{1 / 2}$ \\
$\begin{array}{c}\text { Oscillator } \\
\text { amplitude }\end{array}$ & $10^{-7} / \mathrm{Hz}^{1 / 2}$ & $3 \times 10^{-6} / \mathrm{Hz}^{1 / 2}$ \\
$\begin{array}{c}\text { Oscillator } \\
\text { phase }\end{array}$ & $2 \times 10^{-4} \mathrm{rad} / \mathrm{Hz}^{1 / 2}$ & $10^{-6} \mathrm{rad} / \mathrm{Hz}^{1 / 2}$ \\
\hline
\end{tabular}

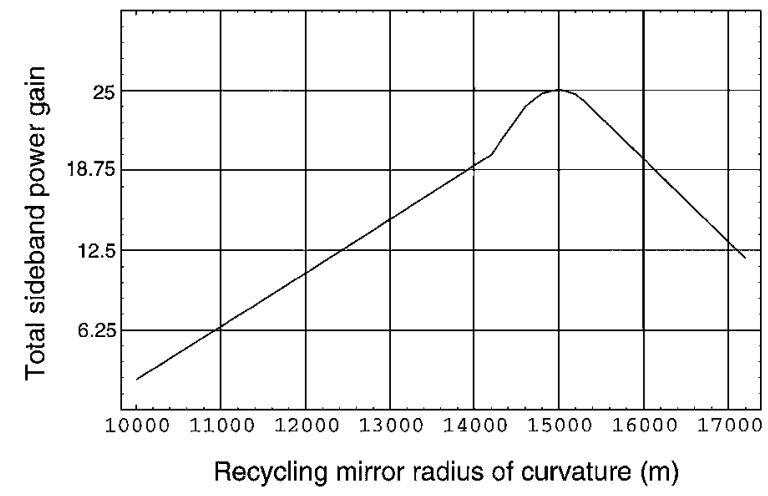

Fig. 2. Sideband gain versus recycling mirror radius of curvature in the presence of end test mass thermal lensing. One can recover the reduced gain at the nominal recycling mirror's curvature $(10 \mathrm{~km})$ by altering the recycling mirror's curvature to compensate for the distorted field. 
scheme as a single one-arm sideband (SOAS) scheme. Using the FFT code, we find that, with no thermal lensing, the SOAS sideband gain in the PRC is 22 with a Michelson asymmetry chosen to maximize the sideband throughput. With the introduction of thermal lensing, the gain falls less than $3 \%$. Changing the radius of curvature from 10 to $13 \mathrm{~km}$ has $\sim 1 \%$ effect: the SOAS gain and throughput are essentially the same. We note that with an impedance-matched carrier the sideband throughput obtained is $70 \%$ because of the additional losses encountered in the arm cavity.

Clearly, using the SOAS scheme overcomes the sideband thermal lensing problem; with the rf sidebands resonating in an arm cavity, the recycling cavity will no longer appear as a degenerate flat mirror cavity. In addition, the system is more stable against recycling cavity geometric errors such as curvature mismatch. FFT runs show that error tolerance on the radii of curvature of the PRC mirrors can be relaxed to $\sim 50 \%$.

The above results were calculated for an input power of $6 \mathrm{~W}$. Further research is needed to determine how much optical power can be supported with this arrangement, but we expect that a factor-of- $\sim 10$ increase should be sustainable. The use of SOAS's would therefore offer significantly greater robustness to an increase in input power.

Furthermore, the rf sidebands in the SOAS scheme propagate in a coupled cavity with a half-linewidth of $\sim 4 \mathrm{~Hz}$ (larger than the SRS carrier linewidth because of the loss that is due to the Michelson asymmetry outcoupling of the sidebands.) As a result, audio noise on both the carrier and the sideband light is strongly filtered above this frequency. Column 2 of Table 1 summarizes the stability requirements on the laser and the rf oscillator for the SOAS scheme for the LIGO to achieve a sensitivity of $10^{-20} \mathrm{~m} / \mathrm{Hz}^{1 / 2}$. We estimated these results by applying the technique of Ref. 6 to the SOAS configuration. Filtering of the rf sidebands leads to a relaxation of the requirements on the audio band frequency and amplitude stability of the laser and the amplitude stability of the RF source by a factor of 30 in comparison with the SRS method. We find a similar factor-of-30 relaxation in the requirement of laser $r f$ intensity noise. However, the required phase stability of the rf oscillator has increased by a factor of 200 . This requirement arises from phase noise generated at the demodulation. In the SRS case, the phase noise of the demodulation is precisely followed by the phase variation of the modulation sideband frequency; however, in the SOAS scheme the sideband noise is filtered, leaving the demodulation noise to produce gw signal noise. This requirement, which is at the performance level of the best currently available commercial oscillators, couples to a sideband frequency offset from an arm cavity resonance of $10 \mathrm{~Hz}$.

An important aspect of the SOAS scheme is that signals are now no longer pure quadrature- or inphase signals. This is so because of the imbalance of the arms of the interferometer for a set of rf sidebands, which mixes the portion of the signal that is due to phase shifts to the rf sidebands into both in-phase and quadrature demodulations. The consequence of this mixing, which depends on interferometer parameters such as cavity coupling and optical gain, are twofold: First, the control matrix derived from the use of one set of $\mathrm{rf}$ sidebands is no longer block diagonal (as in SRS), as common mode and differential signals are now mixed. Second, the demodulation phase that maximizes or minimizes a given of is no longer either 0 or $90 \mathrm{deg}$, and these respective phases are different for every dof. Although it is possible to extract control signals by use of the SOAS scheme, the effects of mixing of common and differential mode signals are not well understood. Block diagonalization may be recovered by introduction of a second set of sidebands that are resonant in the opposite arm, which provides differential mode signals with the same magnitude but opposite sign, and common mode signals with the same sign. We do this by setting $f_{\mathrm{sb} 1}+f_{\mathrm{sb} 2}=(2 m+1) c / 2 l_{-}, f_{\mathrm{sb} 1}+f_{\mathrm{sb} 2}=$ $(n+1) c / l_{+}$, and $f_{\mathrm{sb} 1}-f_{\mathrm{sb} 2}=(p-1 / 2) c / l_{+}$. Taking sums and differences of the demodulated outputs then gives back separation of the common and differential dof's. However, the constraint on arm cavity length matching to ensure signals of the same magnitude is of the order of several micrometers, which will require active control. Finally, the ability to tailor the demodulation phase for a particular dof allows cross coupling to other dof's to be minimized.

In summary, a new optical control scheme for interferometric gw detectors has been proposed, which involves resonating the $\mathrm{rf}$ sidebands in an arm cavity. This procedure achieves a high degree of tolerance to interferometer spatial distortions and noise on the input light.

This work was supported by the National Science Foundation under Cooperative Agreement PHY9210038.

\section{References}

1. A. Abramovici, W. Althouse, R. W. P. Drever, Y. Gursel, S. Kawamura, F. J. Raab, D. Shoemaker, L. Sievers, R. E. Spero, R. E. Vogt, R. Weiss, S. E. Whitcomb, and M. E. Zucker, Science 256, 325 (1992).

2. A. Giazotto, in First Edoardo Amaldi Conference on Gravitational Wave Experiments, E. Coccia, G. Pizella, and F. Ronga, eds. (World Scientific, Singapore, 1995).

3. M. W. Regehr, F. J. Raab, and S. E. Whitcomb, Opt. Lett. 20, 1507 (1995).

4. W. Winkler, K. Danzmann, A. Rudiger, and R. Schilling, Phys. Rev. A 44, 7022 (1991).

5. B. Wilke, N. Uehara, E. K. Gustafson, R. L. Byer, P. King, S. Seel, and R. L. Savage, Opt. Lett. 23, 1704.

6. J. B. Camp, H. Yamamoto, S. E. Whitcomb, and D. E. McClelland, "Analysis of light noise sources in a recycled Michelson interferometer with Fabry-Perot arms," submitted to J. Opt. Soc. Am. A.

7. B. Bochner, "Modelling the performance of interferometric gravitational wave detectors with realistically imperfect optics," Ph.D. dissertation (Massachusetts Institute of Technology, Cambridge, Mass., 1998).

8. R. Flaminio and H. Heitman, Phys. Lett. A 214, 112 (1996). 